\title{
Firmware of the ATLAS Level-0 Endcap Muon Trigger for HL-LHC
}

\author{
Yuki Mitsumori* \\ on behalf of the ATLAS TDAQ Collaboration \\ Graduate School of Science, Nagoya University, \\ Furo-cho, Chikusa-ku, Nagoya, Japan \\ E-mail: ymitsumori@hepl.phys.nagoya-u.ac.jp
}

The design and the status of the development of the Level-0 endcap muon trigger firmware for the ATLAS experiment at the HL-LHC are presented. Reconstruction of muon candidates with an improved momentum resolution is achieved by exploiting all hit data from Thin Gap Chambers (TGCs) to be available at XCVU13P FPGA mounted on the trigger and readout boards. Track segments from muons are reconstructed by a pattern matching algorithm, where the TGC hits are compared with predefined hit patterns. Each predefined hit pattern has position and angle information for associated for the track segment. Implementation of the algorithm with minimal utilisation of the XCVU13P FPGA resource is a major challenge. We achieved resolutions with $1 \mathrm{~cm}$ in position and $4 \mathrm{mrad}$ in angle, which satisfy the requirements, with less than $40 \%$ of the UltraRAM resources for full coverage of TGCs. The performance was evaluated with a post-synthesis simulation with hit inputs from Geant4 full simulation. The implementation was successful without timing violation, by optimising latch circuit locations. The results constitute an important ingredients in the development of the Level-0 endcap muon trigger firmware for HL-LHC.

The Ninth Annual Conference on Large Hadron Collider Physics - LHCP2021

7-12 June 2021

Online

${ }^{*}$ Speaker 


\section{Introduction}

The data acquisition of proton-proton collisions at High-Luminosity LHC (HL-LHC) is planned to start in 2027 [1]. The instantaneous luminosity will increase to $5-7.5 \times 10^{34} \mathrm{~cm}^{-2} \mathrm{~s}^{-1}$ and the total integrated luminosity delivered during ten years of operation will be $3000-4000 \mathrm{fb}^{-1}$. The trigger and data acquisition system of the ATLAS experiment [2] will be upgraded during the long shutdown in 2025-2027 [3]. The new trigger system includes a single level hardware trigger (Level-0 trigger) with a maximum output event rate of $1 \mathrm{MHz}$ and $10 \mu$ s latency. In this report, we describe an overview of the firmware for the muon reconstruction in the endcap region in the Level-0 trigger and the status of the development.

\section{Level-0 endcap muon trigger at HL-LHC}

The Level-0 endcap muon trigger uses signals from Thin Gap Chamber (TGC) on Big Wheel (BW) and several inner detectors, as shown in Fig. 1. TGC is a multiwire proportional chamber that measures two-dimensional muon positions with signals from wires and strips. TGC BW consists of three stations (M1, M2 and M3). The M1 station has three layers. Each of M2 and M3 stations has two layers. They cover pseudorapidity $(\eta)$ range of $1.05<|\eta|<2.4$ behind of the endcap troid.

The current first-level endcap muon trigger identifies muon candidates in simple coincidences with signals from TGC BW then evaluates transverse momentum $\left(p_{\mathrm{T}}\right)$ of the candidates with look-up tables. The new trigger for HL-LHC reconstructs track segments by using all hit information from TGC BW and new $p_{\mathrm{T}}$ is evaluated based on position and angle differences between TGC BW track segments and signals from inner detectors: New Small Wheel (NSW), TGC in the Endcap Inner (EI) station, Resistive Plate Chamber (RPC) in barrel inner station and Tile hadron Calorimeter (TileCal). Signals from Monitored Drift Tube (MDT) chambers around selected muon candidates are processed for more precise $p_{\mathrm{T}}$ evaluation.

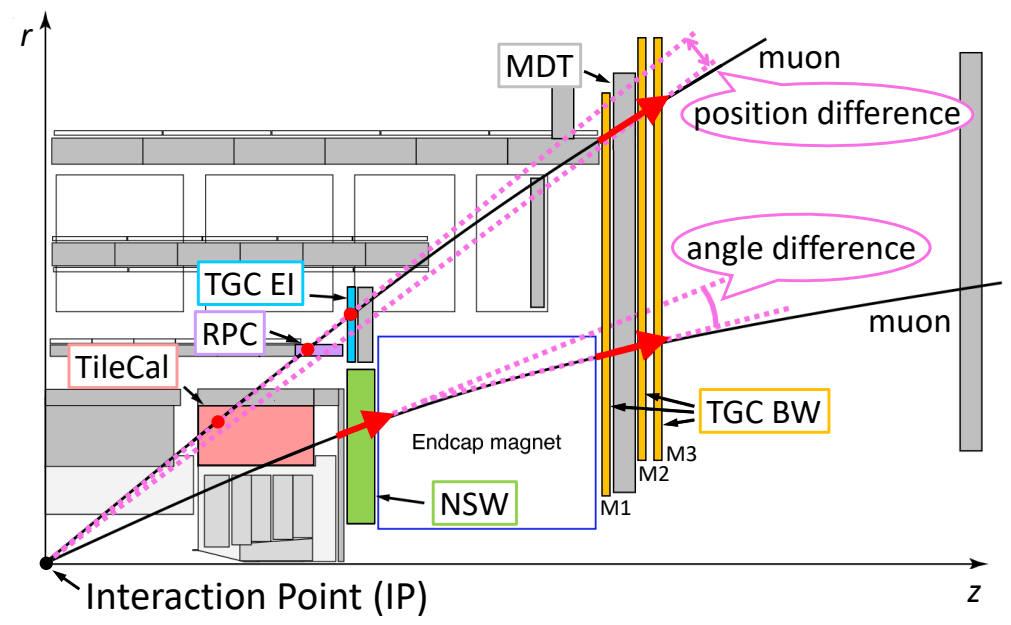

Figure 1: $r-z$ view of the ATLAS muon detectors [3]. The $z$ axis is taken as the beam axis, and the $r$ axis perpendicular to the beam axis. The muon $p_{\mathrm{T}}$ is evaluated in position difference and angle difference obtained with various subdetectors. 


\section{Firmware overview}

The new trigger and readout algorithms will be implemented on a high-end large-scale FPGA (Xilinx XCVU13P [4]). The trigger algorithm consists of four steps shown in Fig. 2. Each step of the algorithm is developed in parallel. We aim to suppress the total resource utilization to less than $50 \%$ in order to avoid timing violations. The target muon reconstruction efficiency is $90 \%$. The readout firmware stores the TGC hits and trigger data until the final result of the Level-0 trigger is received. In the following, the firmware of the wire segment reconstruction and the inner coincidence is focused on.
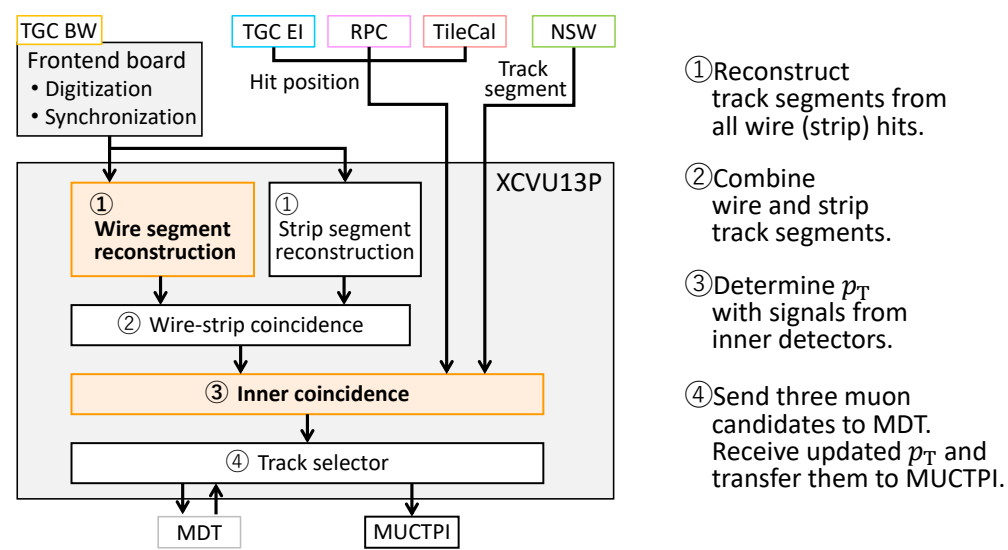

(4) Send three muon candidates to MDT.

Receive updated $p_{\mathrm{T}}$ and transfer them to MUCTPI.

Figure 2: A simplified block diagram of the firmware for the Level-0 endcap muon trigger. Track segments are reconstructed from the signals of TGC BW, and $p_{\mathrm{T}}$ is determined by adding inner detector information.

\section{Wire segment reconstruction firmware}

The wire segment reconstruction firmware reconstructs muon track segments with wire hits of TGC BW in two steps. In the first step, a coincidence is taken in each station then position IDs are obtained as shown in Fig. 3 (Left). Then in the second step, angles of the track segments are obtained by comparing the position IDs on the three stations with hit-pattern lists predefined and stored in RAM of FPGA. The algorithm runs in parallel for the units, defined in Fig. 3 (Right). It also shows the definition of position IDs of the units in each station. In order to suppress the resources, each units is determined to include only the position IDs required for the segment reconstruction for muon with $p_{\mathrm{T}}>4 \mathrm{GeV}$.
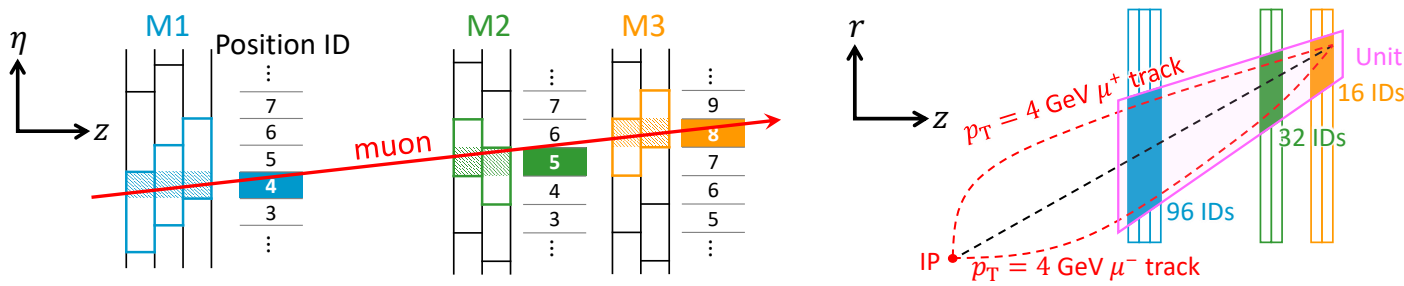

Figure 3: Left: Conceptual view of the station coincidence. Right: Conceptual view of the unit region. 
The firmware for a specific unit was developed and validated with an FPGA mounted an evaluation board, VCU118 [4]. A result of segment reconstruction is shown in Fig. 4. The angular resolution of $2.4 \mathrm{mrad}$ was obtained in the region of $2.13<|\eta|<2.16$. An expanded firmware to whole trigger sector has been successfully implemented without timing violations. Validation for the expanded firmware is ongoing.

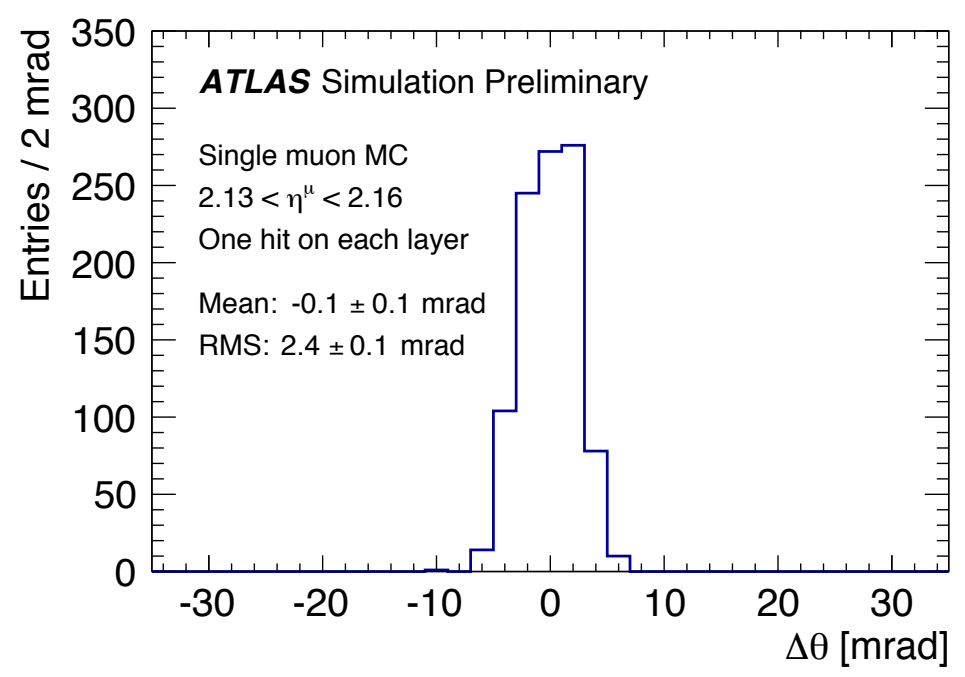

Figure 4: Distribution of angle difference between MC truth track segments and the track segments reconstructed by the developed firmware [5].

\section{Inner coincidence firmware}

For the region $1.05<|\eta|<1.3$, the firmware evaluates $p_{\mathrm{T}}$ with position difference between the TGC BW track segments and hits in TGC EI, RPC and TileCal. For $1.3<|\eta|<2.4$, the firmware evaluates $p_{\mathrm{T}}$ with angle provided by the NSW and position difference between the track segments of TGC BW and NSW. The firmware development is in progress.

\section{Conclusion}

The new ATLAS Level-0 endcap muon trigger for HL-LHC is under development. The new trigger reconstructs muon candidates from all hit information of TGC BW and several other detectors' signals. The firmware for the wire segment reconstruction has been developed in a specific region $(2.13<\eta<2.16)$ and the angular resolution is found to be less than $4 \mathrm{mrad}$, the target. The firmware is being expanded for the full coverage, and the validation is ongoing. The inner coincidence firmware development is also in progress. 


\section{References}

[1] G. Apollinari, I. Béjar Alonso, O. Brüning, P. Fessia, M. Lamont, L. Rossi and L. Tavian, High-Luminosity Large Hadron Collider (HL-LHC) : Technical Design Report V. 0.1, CYRM-2017-004.

[2] ATLAS Collaboration, The ATLAS Experiment at the CERN Large Hadron Collider, JINST 3 (2008) S08003.

[3] The ATLAS Collaboration, Technical Design Report for the Phase-II Upgrade of the ATLAS TDAQ System, CERN-LHCC-2017-020, ATLAS-TDR-029, https://cds. cern.ch/record/2285584.

[4] Xilinx Inc., https://www. xilinx.com/.

[5] The ATLAS Collaboration, LO Muon Trigger Public Results, https://twiki.cern.ch/twiki/bin/view/AtlasPublic/ LOMuonTriggerPublicResults 